07,01

\title{
Влияние температуры деформации на эффект реализации высокой пластичности в ультрамелкозернистом сплаве $\mathrm{Al}-1.5 \mathrm{Cu}$
}

\author{
() А.М. Мавлютов ${ }^{1}$, Т.С. Орлова ${ }^{2}$, Э.Х. Яппарова ${ }^{1}$, Н.А. Еникеев ${ }^{1,3}$, М.Ю. Мурашкин ${ }^{1,3}$ \\ ${ }^{1}$ Санкт-Петербургский государственный университет, \\ Санкт-Петербург, Россия \\ ${ }^{2}$ Физико-технический институт им. А.Ф. Иофффе, \\ Санкт-Петербург, Россия \\ ${ }^{3}$ Институт фризики перспективных материалов, \\ Уфимский государственный авиационный технический университет, \\ Усра, Россия \\ E-mail: a.m.mavlyutov@gmail.com
}

Поступила в Редакцию 10 мая 2021 г.

В окончательной редакции 10 мая 2021 r.

Принята к публикации 13 мая 2021 г.

Впервые исследовано влияние температуры механических испытаний путем одноосного растяжения на эффект пластификации (ЭП) ультрамелкозернистого (УМЗ) сплава $\mathrm{Al}-1.5 \mathrm{Cu}$ (wt.\%). УМ3-структура в материале была сформирована методом интенсивной пластической деформации кручением (ИПДК). Значительное увеличение пластичности УМЗ-сплава с $~ 3$ до 22\% при сохранении высокой предельной прочности (450 MPa) было достигнуто за счет дополнительной деформационно-термической обработки, включающей кратковременный низкотемпературный отжиг и небольшую дополнительную ИПДК. Выявлен температурный интервал реализации ЭП. Показано, что понижение температуры деформации приводит к постепенному уменьшению ЭП и его исчезновению при $-20^{\circ} \mathrm{C}$. Легирование медью привело к значительному сужению со стороны низких температур интервала реализации ЭП по сравнению со случаем УМЗ А1. Обсуждаются возможные причины влияния легирования $\mathrm{Cu}$ на температурную зависимость ЭП.

Ключевые слова: алюминиево-медные сплавы, интенсивная пластическая деформация, ультрамелкозернистая структура, прочность, пластичность, зернограничная сегрегация.

DOI: 10.21883/FTT.2021.10.51409.105

\section{1. Введение}

Сплавы системы $\mathrm{Al}-\mathrm{Cu}$ широко применяются в качестве конструкционных материалов [1]. В связи с этим повышение прочности, пластичности, а также получение комбинации высокой прочности и пластичности в этих сплавах является важной и актуальной задачей. В последние десятилетия активно разрабатываются и используются методы интенсивной пластической деформации (ИПД), которые зарекомендовали себя как хороший способ упрочнения металлов и сплавов за счет создания в них повышенной плотности дефектов, главным образом границ зерен и дислокаций [2]. В процессе ИПД в сплаве формируется ультрамелкозернистая (УМЗ) или нанокристаллическая структура, обеспечивающая повышение прочности в $2-4$ раза по сравнению с прочностью сплавов, подвергнутых традиционным методам деформационно-термической обработки [1]. Однако повышение прочности за счет структурирования методами ИПД приводит к значительному снижению пластичности материала, что ограничивает их практическое применение [2]. Активно развивающимся направлением исследований в области ИПД является изучение влияния постдеформационной обработки УМЗ-материалов на эволюцию микроструктуры и их результирующие свойства, а также установление физической природы и закономерностей взаимосвязи микроструктуры и свойств. Недавно были открыты два принципиально новых явления для УМЗ Al. Было обнаружено, что постдеформационный низкотемпературный отжиг УМ3 Al приводит к дополнительному упрочнению, а последующая небольшая дополнительная деформация к повышению пластичности материала при сохранении высокого уровня прочности $[3,4]$. Повышение пластичности Al после небольшой дополнительной деформации - так называемый эффект пластификации (ЭП) связывался с внесением дислокаций в релаксированную отжигом структуру большеугловых границ зерен [5]. Подобный ЭП наблюдался для сплава Al-1.5 wt.\% Cu в УМЗ-состоянии. Было показано, что низкотемпературный отжиг и небольшая дополнительная деформация после обработки интенсивной пластической деформацией кручением (ИПДК) приводят к повышению пластичности УМЗ-сплава до значений, характерных для крупнозернистого (КЗ) состояния при сохранении высокого уровня прочности [6].

Условия эксплуатации материалов требуют стабильности полученных свойств при изменении температуры окружающей среды. Между тем, известна лишь единственная работа, описывающая влияние температуры испытаний на ЭП в УМЗ Al [7]. Для технически чистого 
УМЗ Al было показано, что эффект повышения пластичности существенным образом зависит от температуры испытаний [7]. Физическая природа механизма реализации высокой пластичности еще полностью не установлена, в связи с этим важным является исследование влияния внешних условий на реализацию данного эффекта. Понижение температуры испытаний может подавить термически активируемые деформационные процессы и может способствовать выявлению ключевых факторов, контролирующих этот эффект.

В настоящей работе мы исследовали влияние температуры деформирования одноосным растяжением на эффект реализации высокой пластичности сплава $\mathrm{Al}-1.5$ wt.\% $\mathrm{Cu}$ в УМЗ-состоянии с различными параметрами структуры.

\section{2. Материал исследования и экспериментальные методики}

В работе был исследован сплав, имеющий следующий химический состав: $1.5 \mathrm{Cu}, 0.3 \mathrm{Ti}, 0.04 \mathrm{Si}, 0.04 \mathrm{Ni}, 0.04 \mathrm{Zn}$, $0.02 \mathrm{Fe}$, ост. Al wt.\%. Исходный материал в виде пластин был предоставлен НИТУ МИСиС (Москва). Предварительно сплав отжигался при $500^{\circ} \mathrm{C}$ в течение $4 \mathrm{~h}$ с последующей закалкой в воду для образования легирующими элементами твердого раствора. Затем для получения УМЗ-структуры из пластин были вырезаны диски диаметром 20 и толщиной $2 \mathrm{~mm}$ и обработаны ИПДК под давлением на 10 оборотов при комнатной температуре. В результате такой обработки были получены диски диаметром 20 и толщиной $\sim 1.0 \mathrm{~mm}$. Степень истинной деформации в области середины радиуса диска составила $\sim 6.6[8]$. Для дальнейших исследований (характеризации микроструктуры, механических и электрических свойств) использовалась преимущественно эта область диска. Часть образцов была подвергнута дополнительной деформационно-термической обработке, включающей в себя отжиг при $150^{\circ} \mathrm{C}$ в течение $1 \mathrm{~h}$ и дополнительную деформацию кручением на 0.25 оборота при тех же давлении и температуре. В дальнейшем образцы после обработки ИПДК обозначены как НРТ, образцы после дополнительной деформационно-термической обработки как НРТ + 150C + 0.25НPТ.

Удельное сопротивление образцов $\left(\rho^{\exp }\right)$ измерялось вихретоковым методом при комнатной температуре, используя измеритель марки ВЭ-27 НЦ/4-5 с относительной погрешностью $\pm 2 \%$ в соответствии с ГОСТ 27333-87. Для каждого образца было сделано не менее 10 измерений электропроводности в области, находящейся на расстоянии $5 \mathrm{~mm}$ от центра диска.

Для испытаний на одноосное растяжение из полученных дисков на электроэрозионном станке были вырезаны образцы в форме лопаток с рабочей частью $5 \times 2 \mathrm{~mm}$ [4]. Одноосное растяжение проводилось на испытательной машине Shimadzu AG-XD Plus при различных температурах испытаний $T_{\mathrm{def}}$ в диапазоне от -80 до $20^{\circ} \mathrm{C}$ в камере, охлаждаемой парами азота. $T_{\mathrm{def}}$ фиксировалась с точностью $\pm 0.5^{\circ} \mathrm{C}$. Скорость деформации составляла $5 \cdot 10^{-4} \mathrm{~s}^{-1}$. Как минимум 3 образца были испытаны для каждого состояния и каждой температуры испытаний. По полученным диаграммам напряжение-деформация были определены условный предел текучести $\left(\sigma_{0.2}\right)$ (напряжение, соответствующее относительному удлинению $0.2 \%)$, предел прочности $\left(\sigma_{\mathrm{UTS}}\right)$ и максимальное относительное удлинение $(\delta)$.

Характеризация микроструктуры была проведена методами просвечивающей электронной микроскопии $($ ПЭМ) и рентгеноструктурного анализа (РСА). Объекты для исследований методом ПЭМ изготавливали струйной полировкой на установке Tenupol-5 в pacтворе $20 \%$ азотной кислоты и $80 \%$ метанола при температуре $-25^{\circ} \mathrm{C}$ и напряжении $15 \mathrm{~V}$. Анализ микроструктуры методом ПЭМ был проведен на микроскопе JEOL JEM 2100 при ускоряющем напряжении $200 \mathrm{kV}$. Средний размер частиц вторичной фазы определяли по результатам измерения не менее 200 частиц с помощью программы „ImageJ“.

Рентгеноструктурный анализ проводился на дифрактометре Bruker D8 DISCOVER в режиме симметричного $\theta-2 \theta$ сканирования с использованием параллельного пучка излучения $\mathrm{Cu} K_{\alpha}(40 \mathrm{kV}, 40 \mathrm{~mA})$. Шаг сканирования по углу $2 \theta$ составил $0.02^{\circ}$, а время экспозиции $-0.5 \mathrm{~s}$. Полнопрофильным моделированием методом Ритвельда с использованием программного обеспечения MAUD определялись значения параметра решетки $a$, средний размер областей когерентного рассеяния $(D$ XRD $)$ и уровень микроискажений кристаллической решетки $\left(\left\langle\varepsilon^{2}\right\rangle^{1 / 2}\right)$. Плотность дислокаций $L_{\mathrm{dis}}$ определяли по формуле [9]:

$$
L_{\mathrm{dis}}=2 \sqrt{3}\left\langle\varepsilon^{2}\right\rangle^{1 / 2} / D_{\mathrm{XRD}} b,
$$

где $b=0.286 \mathrm{~nm}-$ величина вектора Бюргерса дислокации [10].

\section{3. Результаты}

\section{1. Механические и электрические свойства}

На рис. 1 показаны кривые напряжение-деформация для образцов сплава $\mathrm{Al}-1.5 \mathrm{Cu}$ в исходном гомогенизированном состоянии, после ИПДК и после дополнительной обработки, включающей отжиг при $150^{\circ} \mathrm{C}$ в течение $1 \mathrm{~h}$ и ИПДК на 0.25 оборота, полученные при различных температурах испытаний. Как видно, обработка ИПДК приводит к существенному росту предела прочности от $\sim 160$ до $\sim 655 \mathrm{MPa}$, условного предела текучести от $\sim 76$ до $\sim 515 \mathrm{MPa}$, однако пластичность образцов снижается с 21 до $3 \%$ (рис. 1,a). Отжиг при $150^{\circ} \mathrm{C}$ приводит к снижению предела прочности до $\sim 440 \mathrm{MPa}$ и охрупчиванию образцов с невозможностью определить предел текучести. После дополнительной деформации предел прочности материала повышается до $\sim 450 \mathrm{MPa}$, 

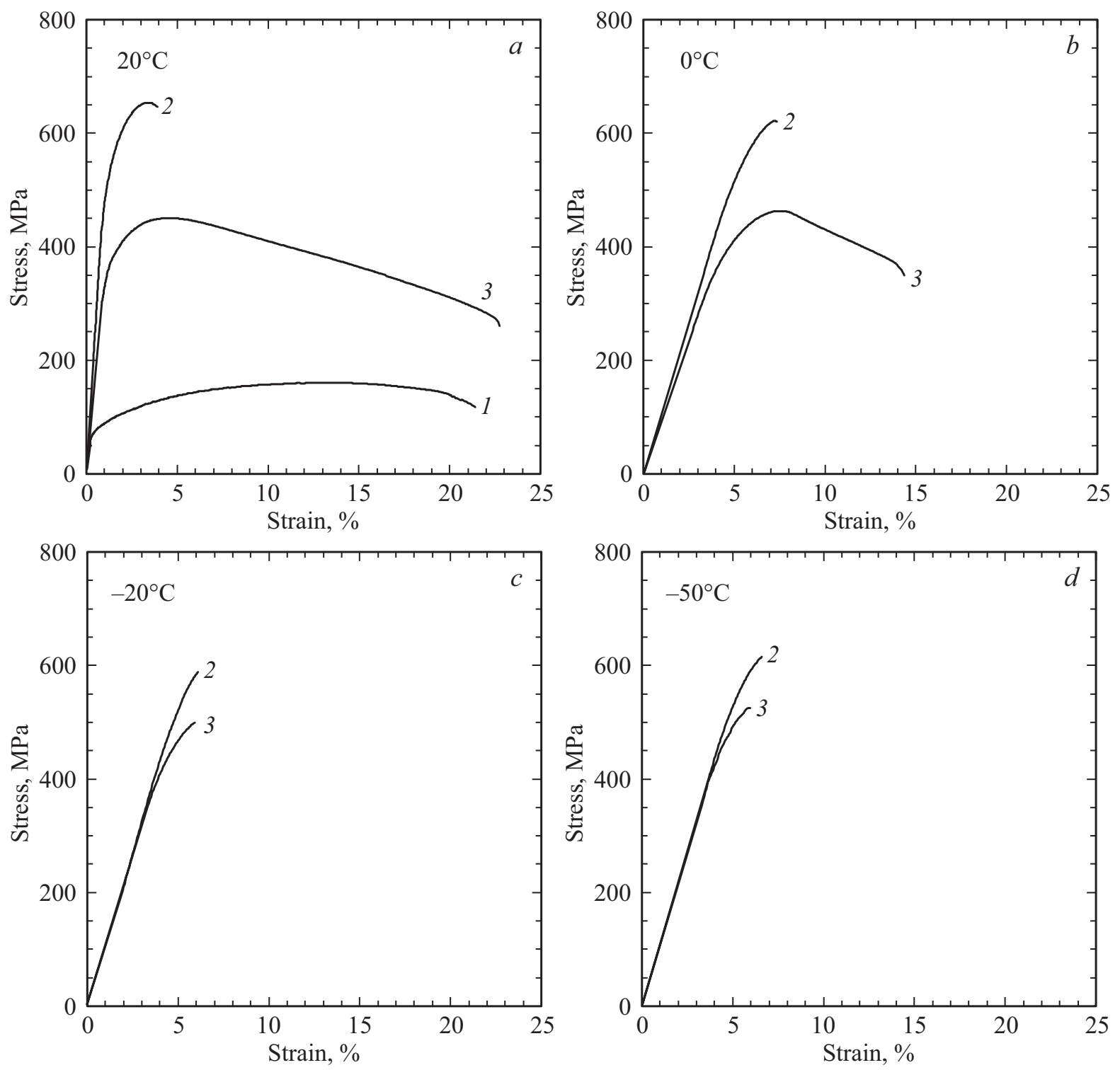

Рис. 1. Диаграммы напряжение-деформация, полученные для образцов сплава $\mathrm{Al}-1.5 \mathrm{Cu}$, в исходном состоянии (кривая 1 ), после ИПДК (кривая 2) и отжига и дополнительной деформации (кривая 3), при различных температурах испытаний на одноосное растяжение $\left(a-20^{\circ} \mathrm{C}, b-0^{\circ} \mathrm{C}, c--20^{\circ} \mathrm{C}, d--50^{\circ} \mathrm{C}\right)$.

условный предел текучести составляет $\sim 345 \mathrm{MPa}$, а пластичность достигает $22 \%$, превышая при этом пластичность исходного образца. Этот результат коррелирует с полученными ранее для данного сплава данными [6] и свидетельствует о том, что дополнительная обработка (отжиг при $150^{\circ} \mathrm{C}$ и ИПДК на 0.25 оборота) позволила реализовать в сплаве ЭП и достичь высокой пластичности при сохранении высоких значений предела прочности и условного предела текучести. Снижение температуры механических испытаний (одноосного растяжения) до 0 и $-10^{\circ} \mathrm{C}$ приводит к тому, что величина ЭП постепенно уменьшается и эффект практически не проявляется при $T_{\text {def }}=-20^{\circ} \mathrm{C}$ (рис. $\left.1, b, c\right)$. А при $T_{\text {def }}=-50^{\circ} \mathrm{C}$ (рис. $\left.1, d\right)$ образцы в обоих состояниях $\mathrm{HPT}$ и $\mathrm{HPT}+150 \mathrm{C}+0.25 \mathrm{HPT}$ становятся практически хрупкими $(\delta \approx 0.5 \%)$. Таким образом, экспериментально получена температурная зависимость ЭП, а также определен температурный интервал его проявления. Таким образом, легирование медью привело к уменьшению температурного интервала проявления ЭП: в технически чистом $\mathrm{Al}$ ЭП наблюдался в области от -50 до $20^{\circ} \mathrm{C}$ [7], тогда как в сплаве $\mathrm{Al}-1.5 \mathrm{Cu}$ только в области -20 до $20^{\circ} \mathrm{C}$ (рис. 2). Кроме того, получены температурные зависимости предела прочности, предела текучести и максимального относительного удлинения для обоих УМ3-состояний НРТ и НРТ $+150 \mathrm{C}+0.25 \mathrm{HPT}$ во всем температурном диапазоне испытаний (рис. 2 и табл. 1).

В исходном состоянии удельное сопротивление сплава $\left(\rho^{\exp }\right)$ составляет $\sim 39.7 \mathrm{n} \Omega \mathrm{m}$, в результате обработки ИПДК $\rho^{\exp }$ увеличивается до $\sim 41.3 \mathrm{n} \Omega \mathrm{m}$ (табл. 2). От- 

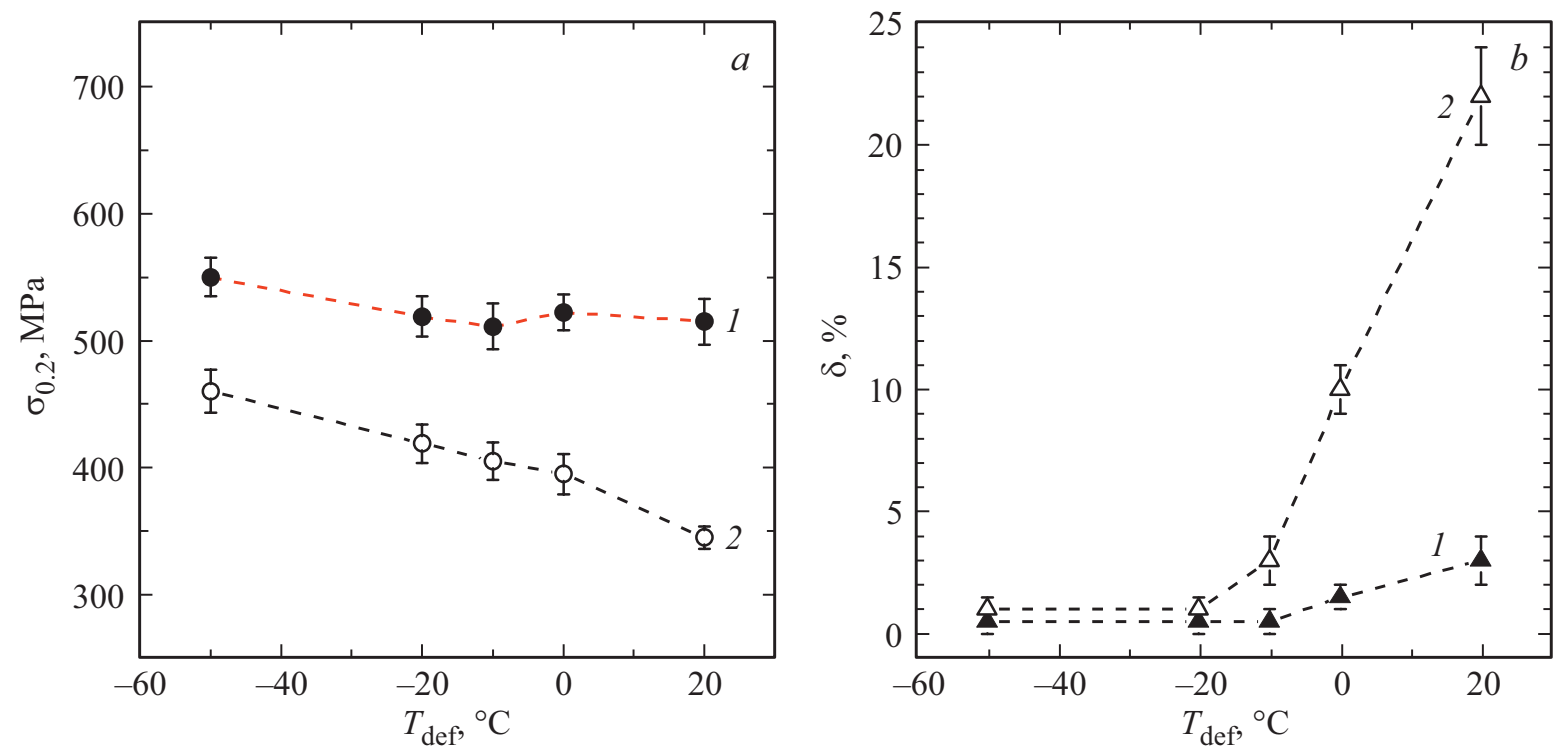

Рис. 2. Зависимости условного предела текучести $(a)$ и удлинения до разрушения $(b)$ для образцов сплава $\mathrm{Al}-1.5 \mathrm{Cu}$ после ИПДК (кривая 1) и отжига и дополнительной деформации (кривая 2) от температуры испытаний на одноосное растяжение.

жиг и дополнительная деформация приводят к снижению удельного сопротивления до $\sim 38.2$ и $\sim 37.7 \mathrm{n} \Omega \mathrm{m}$ соответственно. Увеличение удельного сопротивления после обработки ИПДК может быть связано с измельчением зеренной структуры, а также с созданием неравновесной структуры в границах зерен [11]. С другой стороны, очищение алюминиевой матрицы в результате распада твердого раствора и преципитации способствует снижению удельного сопротивления [12]. Более подробно эволюция микроструктуры в корреляции с изменением механических и электрических свойств будет рассмотрена ниже.

\section{2. Эволюция микроструктуры}

В исходном гомогенизированном состоянии образец имел КЗ-структуру со средним размером зерна

Таблица 1. Механические свойства сплава $\mathrm{Al}-1.5 \mathrm{Cu}$ при различных температурах деформации ( $\sigma_{0.2}-$ условный предел текучести, $\sigma_{\mathrm{UTS}}$ - предел прочности, $\delta$ - относительное удлинение до разрушения)

\begin{tabular}{|c|c|c|c|c|}
\hline $\begin{array}{c}\text { Температура } \\
\text { деформации, }{ }^{\circ} \mathrm{C}\end{array}$ & Состояние & $\begin{array}{l}\sigma_{0.2} \\
\mathrm{MPa}\end{array}$ & $\begin{array}{l}\sigma_{\text {UTS }} \\
\mathrm{MPa}\end{array}$ & $\delta, \%$ \\
\hline \multirow{2}{*}{-80} & HPT & $640 \pm 18$ & $666 \pm 16$ & $\sim 0.5$ \\
\hline & $\mathrm{HPT}+150 \mathrm{C}+0.25 \mathrm{HPT}$ & $482 \pm 13$ & $521 \pm 14$ & $\sim 0.5$ \\
\hline \multirow{2}{*}{-50} & HPT & $550 \pm 15$ & $582 \pm 14$ & $\sim 0.5$ \\
\hline & $\mathrm{HPT}+150 \mathrm{C}+0.25 \mathrm{HPT}$ & $460 \pm 17$ & $521 \pm 11$ & $\sim 1$ \\
\hline \multirow{2}{*}{-20} & HPT & $519 \pm 16$ & $570 \pm 15$ & $\sim 0.5$ \\
\hline & $\mathrm{HPT}+150 \mathrm{C}+0.25 \mathrm{HPT}$ & $419 \pm 15$ & $502 \pm 15$ & $\sim 1$ \\
\hline \multirow{2}{*}{-10} & HPT & $511 \pm 18$ & $590 \pm 17$ & $\sim 1$ \\
\hline & $\mathrm{HPT}+150 \mathrm{C}+0.25 \mathrm{HPT}$ & $405 \pm 15$ & $498 \pm 13$ & $3 \pm 1$ \\
\hline \multirow{2}{*}{0} & HPT & $522 \pm 14$ & $611 \pm 13$ & $\sim 1.5$ \\
\hline & $\mathrm{HPT}+150 \mathrm{C}+0.25 \mathrm{HPT}$ & $395 \pm 16$ & $473 \pm 9$ & $10 \pm 1$ \\
\hline \multirow{3}{*}{20} & HPT & $515 \pm 18$ & $655 \pm 17$ & $3 \pm 1$ \\
\hline & $\mathrm{HPT}+150 \mathrm{C}$ & - & $440 \pm 11$ & - \\
\hline & $\mathrm{HPT}+150 \mathrm{C}+0.25 \mathrm{HPT}$ & $345 \pm 9$ & $450 \pm 9$ & $22 \pm 2$ \\
\hline
\end{tabular}


$a$
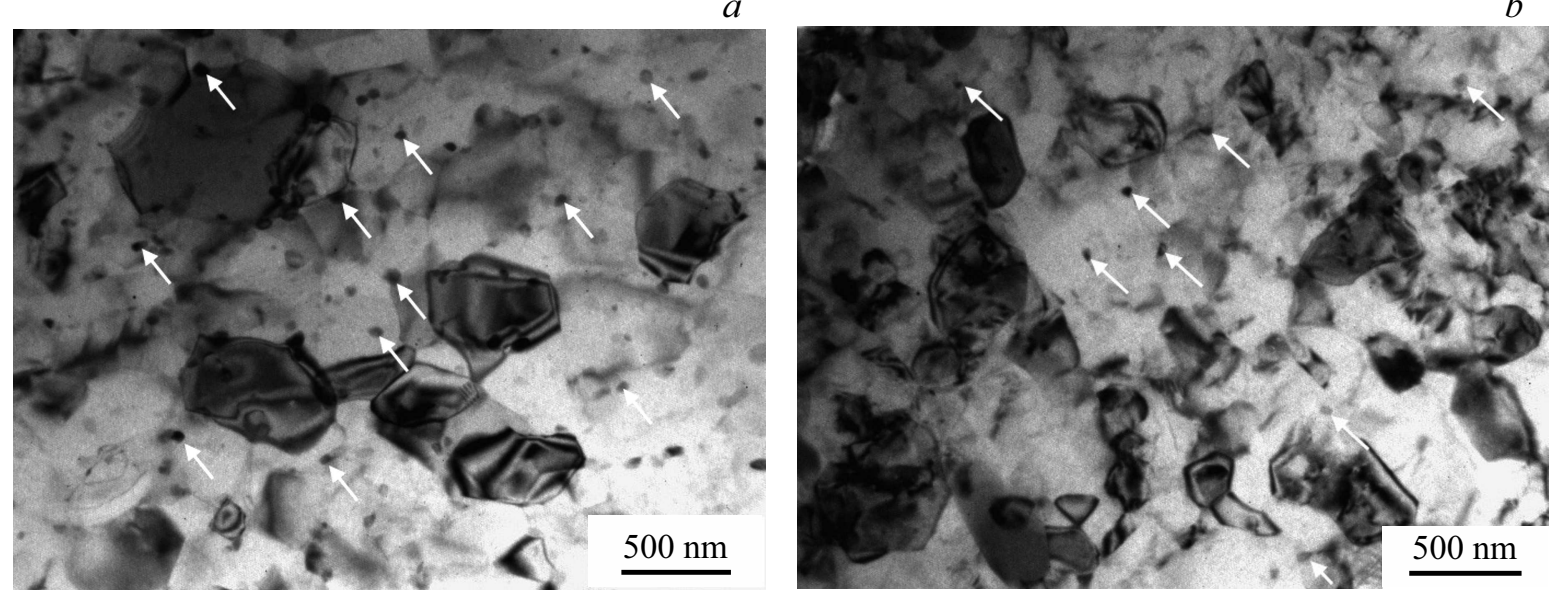

$c$
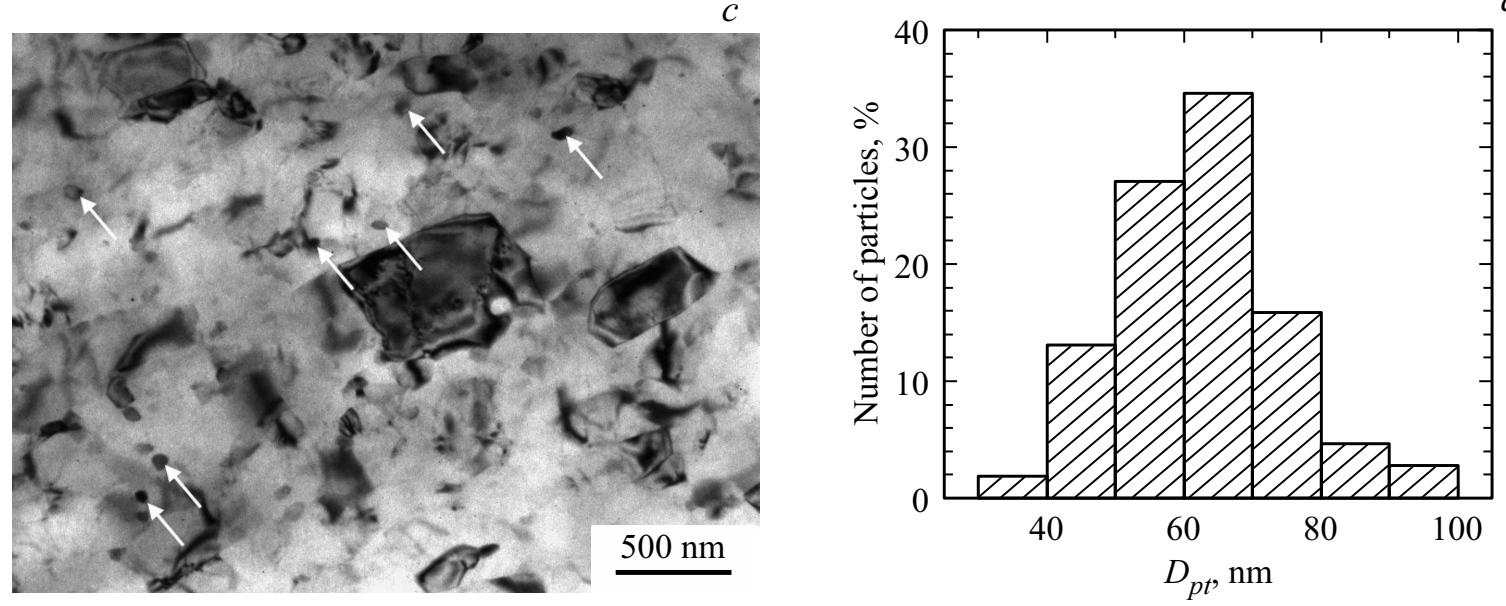

Рис. 3. ПЭМ-изображения структуры УМЗ-сплава $\mathrm{Al}-1.5 \mathrm{Cu}$ после ИПДК $(a)$, отжига $(b)$, отжига и дополнительной деформации $(c)$ и распределение частиц вторичной фазы по размерам для образца после ИПДК $(d)$. Стрелками указаны частицы вторичной фазы.

$d_{a v} \approx 60 \mu \mathrm{m}$. В результате обработки ИПДК в сплаве сформировалась УМЗ-структура со средним размером зерна $d_{a v} \approx 320 \mathrm{~nm}$ (рис. 3, $a$, табл. 3). Последующий отжиг УМЗ-образцов и дополнительная деформация не привели к существенному изменению среднего размера зерна (рис. 3,b,c, табл. 3). Как было показано для технически чистого $\mathrm{Al}$ [13], сплавов систем $\mathrm{Al}-\mathrm{Zr}[14,15], \mathrm{Al}-\mathrm{Mg}-\mathrm{Si}[16,17]$, низкотемпературный отжиг УМЗ-образцов или небольшая дополнительная деформация не приводят к существенному изменению среднего размера зерна, но, по-видимому, влияют на тонкую структуру ГЗ [5]. После обработки ИПДК в структуре присутствует большое количество частиц вторичной фазы сферической формы (рис. 3,a). Средний диаметр частиц составляет $\sim 55 \mathrm{~nm}$, расстояние между частицами $\sim 300 \mathrm{~nm}$ (табл. 3). Как показывают результаты PCA (рис. 4) данные частицы являются стабильной модификацией вторичной фазы $\mathrm{Al}_{2} \mathrm{Cu}$. По данным PCA параметр решетки сплава в КЗ-состоянии составляет $4.0475 \AA$, который увеличивается в результате обработки ИПДК до 4.0486, что свидетельствует об очище-

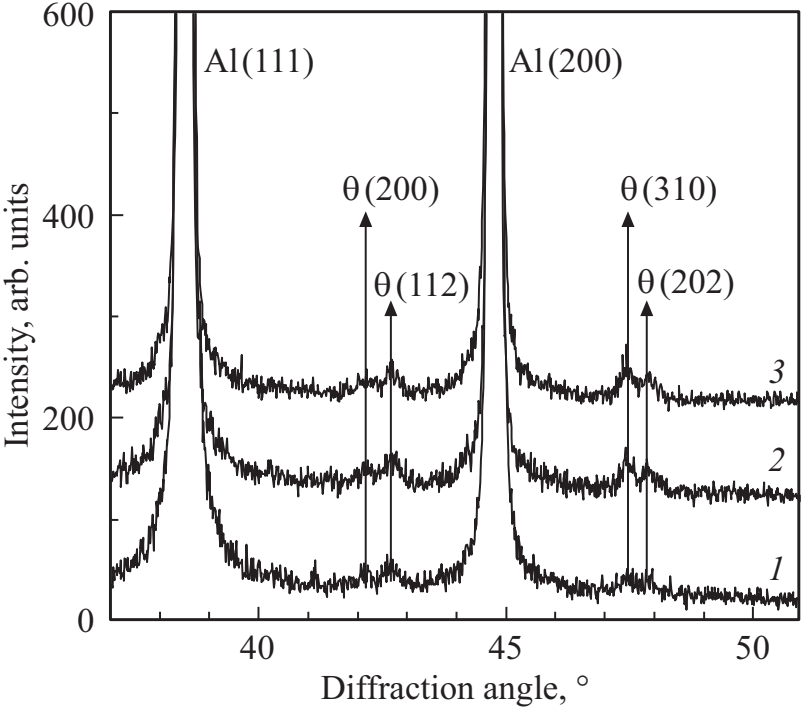

Рис. 4. Рентгенограммы образцов УМ3-сплава $\mathrm{Al}-1.5 \mathrm{Cu}$ после ИПДК (1), отжига (2) и отжига и дополнительной деформации (3). 
нии алюминиевой матрицы от растворенных атомов $\mathrm{Cu}$ [1]. После отжига параметр решетки увеличивается до $4.0495 \AA$, что свидетельствует о еще большем очищении алюминиевой матрицы от растворенных атомов $\mathrm{Cu}$. Последующая дополнительная деформация практически не изменяет параметр решетки, он составляет $4.0497 \AA$. Отжиг при $150^{\circ} \mathrm{C}$ приводит к увеличению размеров ОКР и снижению уровня микронапряжений. Согласно соотношению (1) и данным РСА (табл. 3) плотность дислокаций снижается в результате отжига в $\sim 3$ раза. Дополнительная деформация приводит к фрагментации зерен, росту микронапряжений и, соответственно, увеличению плотности дислокаций до уровня, характерного для состояния НРТ (табл. 3). Подобная тенденция изменения плотности дислокаций ранее наблюдалась и для УМЗ технически чистого $\mathrm{Al}$ [5].

\section{4. Обсуждение полученных результатов}

Проведенная характеризация микроструктуры позволяет провести анализ вкладов различных структурных элементов в предел текучести и удельное сопротивление материала и сравнить их с полученными экспериментальными данными.

1. Границы зерен в поликристаллах выступают барьерами для движения скользящих дислокаций, поскольку при переходе через ГЗ изменяется ориентировка плоскости скольжения. Около межзеренной границы возникает скопление дислокаций, упругие поля которых приводят к испусканию новой дислокации в соседнем зерне [18]. Согласно соотношению Холла-Петча $[19,20]$ вклад зернограничного упрочнения в предел текучести может быть определен как

$$
\sigma_{\mathrm{GB}}=K_{y} d_{a v}^{-1 / 2},
$$

где $K_{y}=0.1 \mathrm{MPa} \cdot \mathrm{m}^{1 / 2}$ - коэффициент Холла-Петча.

2. Деформационное (дислокационное) упрочнение, обусловленное междислокационными взаимодействиями, которые приводят к снижению мобильности дислокаций, можно оценить согласно соотношению Тэйлоpa $[21]$ :

$$
\sigma_{\mathrm{dis}}=M \alpha G b \sqrt{L_{\mathrm{dis}}},
$$

где $M=3.06$ - фактор Тейлора (ориентационный множитель) [22], $\alpha=0.33$ - коэффициент междислокационного взаимодействия [23], $G=26 \mathrm{GPa}-$ модуль сдвига.

3. Движению дислокаций препятствуют примесные и легирующие атомы, находящиеся в твердом растворе. Энергия взаимодействия дислокации с примесным атомом зависит от размерного несоответствия радиусов примесного атома и атома матричного элемента $[24,18]$. Согласно [25] твердорастворное упрочнение определяется уравнением

$$
\sigma_{S S}=k_{\mathrm{Cu}} C_{\mathrm{Cu}}^{2 / 3}+k_{\mathrm{Ti}} C_{\mathrm{Ti}}^{2 / 3}
$$

где $\quad k_{\mathrm{Cu}}=46.4 \mathrm{MPa} /$ wt.\% ${ }^{2 / 3} \quad[26], \quad k_{\mathrm{Ti}}=$ $25.6 \mathrm{MPa} /$ wt.\% $\%^{2 / 3}[26]$ - соответственно коэффициенты, определяющие взаимодействие дислокации с атомами $\mathrm{Cu}$ и Ti; $C_{\mathrm{Cu}}, C_{\mathrm{Ti}}($ wt.\%) - соответственно концентрация атомов $\mathrm{Cu}$ и Ті в твердом растворе.

4. Торможение дислокаций дисперсными частицами $\mathrm{Al}_{2} \mathrm{Cu}$, может быть определено согласно соотношению Орована [27,28]:

$$
\sigma_{O r}=\frac{0.4 M G b}{\pi L_{p t} \sqrt{1-v}} \ln \frac{D_{p t}}{b},
$$

где: $L_{p t}-$ расстояние между частицами, $D_{p t}$ - диаметр частиц, $v=0.35$ коэффициент Пуассона.

Согласно [21,29-31] предел текучести материала является суперпозицией вкладов различных механизмов упрочнения

$$
\sigma_{y}=\sigma_{0}+\sigma_{G B}+\sigma_{\mathrm{dis}}+\sigma_{S S}+\sigma_{O r},
$$

где $\sigma_{0}-$ минимальное касательное напряжение, необходимое для скольжения дислокации в идеальном кристалле (напряжение Пайерлса-Набарро) [24], являющееся константой материала (для $\mathrm{Al} \sigma_{0}=10 \mathrm{MPa}$ ).

Аналогично удельное сопротивление металла согласно правилу Матиссена [32] равно сумме вкладов от различных механизмов рассеяния заряда, каждый из которых не влияет на другие

$$
\begin{aligned}
\rho^{\text {met }}= & \rho^{\text {pure }}+N_{V} \Delta \rho^{\text {vac }}+L_{\mathrm{dis}} \Delta \rho^{\mathrm{dis}}+S_{G B} \Delta \rho^{G B} \\
& +C_{\mathrm{Cu}} \Delta \rho^{\mathrm{Cu}}+C_{\mathrm{Ti}} \Delta \rho^{\mathrm{Ti}},
\end{aligned}
$$

где $\rho^{\text {pure }}=27 \mathrm{n} \Omega \mathrm{m} \quad-$ удельное $\quad$ сопротивление чистого монокристаллического Al при КТ [33], $\Delta \rho^{\mathrm{vac}}=26 \mathrm{n} \Omega \mathrm{m} / \mathrm{at} . \% \quad[34], \Delta \rho^{\mathrm{dis}}=2.7 \cdot 10^{-25} \Omega \mathrm{m}^{3} \quad[35]$, $\Delta \rho^{G B}=2.6 \cdot 10^{-16} \Omega \mathrm{m}^{2}[35]$ - вклады от единичной концентрации вакансий, единичных плотностей дислокаций и ГЗ, соответственно, $\Delta \rho^{\mathrm{Cu}}=3.3 \mathrm{n} \Omega \mathrm{m} / \mathrm{at} . \%$ [1], $\Delta \rho^{\mathrm{Ti}}=25 \mathrm{n} \Omega \mathrm{m} / \mathrm{at} . \% \quad[1] \quad$ - соответственно, вклады от единичной концентрации атомов $\mathrm{Cu}$ и $\mathrm{Ti}$ в твердом растворе, $N_{V}($ at.\%) - концентрация вакансий, $S_{G B}\left(\mathrm{~m}^{-1}\right)$ - объемная плотность ГЗ.

Известно, что вклад от вакансий в электросопротивление алюминия даже после больших пластических деформаций пренебрежимо мал, поскольку вакансии быстро аннигилируют [36]. Вклад в удельное сопротивление от единичной плотности ГЗ существенно зависит от структуры ГЗ: степени их неравновесности [11], угла разориентировки [37], наличия зернограничных сегрегаций $[10]$.

Используя параметры микроструктуры (табл. 3) и соотношения (2)-(7), была проведена оценка вкладов от различных механизмов упрочнения в предел текучести и механизмов рассеяния заряда в удельное сопротивление материала, полученные оценки $\sigma_{y}$ и $\rho^{\text {met }}$ в сравнении с экспериментальными данными $\sigma_{0.2}$ и $\rho^{\exp }$ представлены в табл. 2 и 4. Для К3-состояния оценки вкладов показали 
Таблица 2. Оценка вкладов различных параметров микроструктуры в удельное сопротивление в сравнении с экспериментальными значениями удельного сопротивления образцов сплава $\mathrm{Al}-1.5 \mathrm{Cu}$ в различных состояниях

\begin{tabular}{l|c|c|c|c|c|c|c}
\hline \multicolumn{1}{c|}{ Состояние } & $\rho^{\text {exp }}, \mathrm{n} \Omega \mathrm{m}$ & $\rho^{\text {pure }}, \mathrm{n} \Omega \mathrm{m}$ & $L_{\mathrm{dis}} \Delta \rho^{\mathrm{dis}}, \mathrm{n} \Omega \mathrm{m}$ & $S_{G B} \Delta \rho^{G B}, \mathrm{n} \Omega \mathrm{m}$ & $C_{\mathrm{Cu}} \Delta \rho^{\mathrm{Cu}}, \mathrm{n} \Omega \mathrm{m}$ & $C_{\mathrm{Ti}} \Delta \rho^{\mathrm{Ti}}, \mathrm{n} \Omega \mathrm{m}$ & $\rho^{\mathrm{met}}, \mathrm{n} \Omega \mathrm{m}$ \\
\hline Исходное & $39.7 \pm 0.8$ & 27.0 & $<0.01$ & $<0.01$ & 4.9 & 7.5 & 39.4 \\
НРТ & $41.3 \pm 0.8$ & 27.0 & $<0.01$ & 2.6 & 0.9 & 7.5 & 38.0 \\
НРТ+150С & $38.2 \pm 0.8$ & 27.0 & $<0.01$ & 2.6 & 0.9 & 7.5 & 38.0 \\
НРТ+150С+0.25НРТ & $37.7 \pm 0.8$ & 27.0 & $<0.01$ & 2.6 & 0.9 & 7.5 & 38.0
\end{tabular}

Таблица 3. Результаты анализа микроструктуры сплава $\mathrm{Al}-1.5 \mathrm{Cu}$ в различных состояниях $\left(d_{a v}-\right.$ средний размер зерна, $D_{p t}-$ средний размер частиц вторичной фазы, $L_{p t}-$ среднее расстояние между частицами вторичной фазы, $a-$ параметр решетки, $D_{\mathrm{XRD}}$ - размер областей когерентного рассеяния, $\left\langle\varepsilon^{2}\right\rangle^{1 / 2}-$ уровень микроискажений кристаллической решетки, $L_{\mathrm{dis}}-$ плотность дислокаций)

\begin{tabular}{l|c|c|c|c|c|c|c}
\hline \multicolumn{1}{c|}{ Состояние } & $d_{a v}, \mu \mathrm{m}$ & $D_{p t}, \mathrm{~nm}$ & $L_{p t}, \mathrm{~nm}$ & $a, \AA$ & $D_{\text {ХRD, }} \mathrm{nm}$ & $\left\langle\varepsilon^{2}\right\rangle^{1 / 2}$ & $L_{\text {dis }} \times 10^{13}, \mathrm{~m}^{-2}$ \\
\hline Исходное & $60 \pm 10$ & - & - & $4.0475 \pm 0.0002$ & - & - & - \\
НРТ & $0.3 \pm 0.05$ & $57 \pm 5$ & $280 \pm 20$ & $4.0486 \pm 0.0001$ & $186 \pm 4$ & $5.0 \pm 0.03$ & 3.3 \\
НРТ+150С & $0.3 \pm 0.05$ & $54 \pm 8$ & $300 \pm 40$ & $4.0495 \pm 0.00003$ & $385 \pm 2$ & $3.3 \pm 0.03$ & 1.0 \\
НРТ+150С+0.25НРТ & $0.3 \pm 0.05$ & $57 \pm 8$ & $340 \pm 55$ & $4.0497 \pm 0.00005$ & $197 \pm 3$ & $4.7 \pm 0.06$ & 2.9
\end{tabular}

Таблица 4. Оценка вкладов различных параметров микроструктуры в предел текучести в сравнении с экспериментальными данными изменения условного предела текучести образцов сплава $\mathrm{Al}-1.5 \mathrm{Cu}$ в различных состояниях

\begin{tabular}{l|c|c|c|c|c|c|c|c}
\hline \multicolumn{1}{c|}{ Состояние } & $\sigma_{0}, \mathrm{MPa}$ & $\sigma_{G B}, \mathrm{MPa}$ & $\sigma_{\text {dis }}, \mathrm{MPa}$ & $\sigma_{S S}(\mathrm{Cu}), \mathrm{MPa}$ & $\sigma_{S S}(\mathrm{Ti}), \mathrm{MPa}$ & $\sigma_{O r}, \mathrm{MPa}$ & $\sigma_{y}, \mathrm{MPa}$ & $\sigma_{0.2}, \mathrm{MPa}$ \\
\hline Исходное & 10 & 13 & - & 61 & 12 & - & $\mathbf{9 6}$ & $\mathbf{7 6} \pm \mathbf{1 2}$ \\
HРТ & 10 & 180 & 43 & 35 & 12 & 63 & $\mathbf{3 4 3}$ & $\mathbf{5 1 5} \pm \mathbf{1 8}$ \\
HРТ $+150 \mathrm{C}$ & 10 & 180 & 24 & 35 & 12 & 63 & $\mathbf{3 2 4}$ & - \\
НРТ $+150 \mathrm{C}+0.25 \mathrm{HPT}$ & 10 & 180 & 40 & 35 & 12 & 63 & $\mathbf{3 4 0}$ & $\mathbf{3 4 5} \pm \mathbf{9}$
\end{tabular}

хорошее соответствие с полученным экспериментальным значением $\sigma_{0.2}$ с учетом того, что размер зерна в КЗ состоянии варьируется в довольно широких пределах. Однако для состояния НРТ имеет место несоответствие теоретических оценок с экспериментально полученными значениями. Данное несоответствие может быть обусловлено наличием дополнительных, характерных только для УМЗ-материала, механизмов упрочнения. В работе [38] для сплава $\mathrm{Al}-\mathrm{Cu}$ с близким содержанием $\mathrm{Cu}$ (0.83 at.\%) было показано, что $\mathrm{Cu}$ сегрегирует в ГЗ в процессе ИПДК с превышением концентрации $\mathrm{Cu}$ в ГЗ в несколько раз по сравнению с зерном. Согласно [39], сегрегации $\mathrm{Cu}$ и $\mathrm{Mg}$ на ГЗ в сплаве системы $\mathrm{Al}-\mathrm{Mg}-\mathrm{Si}$, формирующиеся в процессе ИПДК, приводят к дополнительному упрочнению материала. Полученная разница между теоретически рассчитанным пределом текучести $\sigma_{y}$ и экспериментально определенным $\sigma_{0.2}$, наиболее вероятно, связана с образованием сегрегаций $\mathrm{Cu}$ на ГЗ. Обработка ИПДК приводит к формированию неравновесных ГЗ в Al [2], характеризующихся повышенной плотностью дефектов и избыточной энергией [2]. Сегрегация $\mathrm{Cu}$ в ГЗ может приводить к понижению степени неравновесности ГЗ [2]. В работе [39] указывается на возможность формирования мельчайших примесных кластеров, в том числе медных в сплаве системы $\mathrm{Al}-\mathrm{Mg}-\mathrm{Si}$ под действием ИПДК, которые тоже вносят вклад в упрочнение.

Оценки вкладов в удельное сопротивление для образца в исходном состоянии также показали хорошее соответствие с экспериментальными данными (табл. 2). Для состояния НРТ характерно более высокое экспериментально определенное значение удельного сопротивления, что также указывает на присутствие дополнительного механизма рассеяния заряда. Для образцов после отжига и дополнительной деформации оценки вкладов в удельное сопротивление находятся в хорошем соответствии с экспериментальными значениями (табл. 2).

Описанная выше возможная сегрегация $\mathrm{Cu}$ на ГЗ несомненно будет приводить к увеличению их удельного сопротивления. В процессе дальнейшего отжига при $150^{\circ} \mathrm{C}$ может происходить дальнейшая релаксация неравновесных ГЗ за счет аннигиляции дефектов и сегрегационных процессов, которые могут сопровождаться образованием примесных кластеров или преципитатов на ГЗ [38]. Понижение электросопротивления в состоянии после отжига, наиболее вероятно, связано с 
дальнейшим очищением матрицы алюминия от примесных элементов, что согласуется с данными РСА по изменению параметра решетки.

Пластичность образцов в нанокристаллических и УМЗ-сплавах при комнатной температуре реализуется за счет механизмов испускания зернограничных дислокаций в тело зерна, движения дислокаций в зерне, захвата дислокаций противоположной границей и переползанием захваченной дислокации внутри границы [5]. Согласно модели, объясняющей ЭП в СР Al [5], дополнительная деформация на 0.25 оборота приводит к внесению решеточных дислокаций в релаксированную отжигом структуру границ зерен, которые под действием внешней нагрузки могут образовывать скопления скользящих зернограничных дислокаций у тройных стыков и облегчать испускание большого количества дислокаций из тройных стыков.

Согласно работе [7], уменьшение ЭП с понижением температуры в УМ3 Al связывается с тем, что скольжение внесенных зернограничных дислокаций, формирующих их скопление у тройных стыков, является термоактивированным процессом. При понижении температуры деформации $T_{\text {def }}$ подвижность этих дислокаций понижается экспоненциально и при температурах ниже некоторой критической недостаточно времени для формирования скопления дислокаций, необходимого для их эмиссии. Наличие сегрегаций/преципитатов на ГЗ и в тройных стыках будет дополнительно затруднять образование скоплений дислокаций у тройных стыков, поэтому при каждой конкретной температуре деформации потребуется более длительное время для их формирования в границах с сегрегациями по сравнению с ГЗ без сегрегаций. С этим, наиболее вероятно, и связано уменьшение температурного интервала существования ЭП в сплаве $\mathrm{Al}-1.5 \mathrm{Cu}$ по сравнению с технически чистым Al. Таким образом, результаты, полученные для сплава $\mathrm{Al}-1.5 \mathrm{Cu}$ находятся в хорошем согласии с теоретическими моделями, предложенными в $[5,7]$ и объясняющими ЭП в $\mathrm{Al}$.

\section{5. Заключение}

В настоящей работе методом ИПДК была сформирована УМ3-структура в сплаве $\mathrm{Al}-1.5 \mathrm{wt} . \% \mathrm{Cu}$. УМЗ материал демонстрирует высокие значения предела прочности $\sim 655 \mathrm{MPa}$ и условного предела текучести $\sim 515 \mathrm{MPa}$, но имеет низкую пластичность $\sim 3 \%$. Результаты характеризации микроструктуры и анализ вкладов от различных параметров микроструктуры в результирующие значения предела текучести и удельного сопротивления показывают, что повышение прочности УМЗ-образцов обусловлено измельчением зеренной структуры, формированием частиц вторичной фазы, повышением плотности дислокаций, а также дополнительными механизмами, такими как зернограничные сегрегации и возможное образование примесных кластеров.
В результате дополнительной термомеханической обработки, включающей кратковременный низкотемпературный отжиг и последующую небольшую деформацию кручением, достигнуто существенное повышение пластичности УМЗ-образцов до $\sim 22 \%$, при сохранении высоких значений предела прочности $450 \mathrm{MPa} \mathrm{и}$ условного предела текучести $345 \mathrm{MPa}$. Данный эффект, наиболее вероятно, обусловлен повышением плотности дислокаций в структуре в $\sim 3$ раза, поскольку дополнительная обработка не приводит к существенным изменениям среднего размера зерна и распределения частиц вторичной фазы. Впервые исследовано влияние температуры деформации на эффект пластификации и установлены критическая температура и температурный диапазон реализации данного эффекта.

Полученные результаты демонстрируют высокий потенциал для практического применения УМЗ-сплавов системы $\mathrm{Al}-\mathrm{Cu}$, полученных методом ИПД.

\section{Финансирование работы}

Авторы А.М.М. и Э.Х.Я. благодарят Российский научный фонд (грант № 19-79-00114) за финансовую поддержку работы.

Рентгеноструктурные исследования проведены с использованием оборудования Ресурсного центра Научного парка СПбГУ „Рентгенодифракционные методы исследования“.

\section{Конфликт интересов}

Авторы заявляют, что у них нет конфликта интересов.

\section{Список литературы}

[1] L.F. Mondolfo. Aluminum Alloys: Structure and Properties. Elsevier. (2013). $982 \mathrm{c}$.

[2] R.Z. Valiev, A.P. Zhilyaev, T.G. Langdon. Bulk Nanostructured Materials: Fundamentals and Applications, John Wiley \& Sons. (2013).

[3] X. Huang, N. Hansen, N. Tsuji. Science 312, 249 (2006).

[4] А.М. Мавлютов, Т.А. Латынина, М.Ю. Мурашкин, Р.3. Валиев, Т.С. Орлова. ФТТ 59, 10, 1949 (2017).

[5] T.S. Orlova, N.V. Skiba, A.M. Mavlyutov, R.Z. Vaiev, M.Y. Murashkin, M.Y. Gutkin. Rev. Adv. Mater. Sci. 57, 224 (2018).

[6] А.М. Мавлютов, Т.С. Орлова. Э.Х. Яппарова. ПЖТФ 46, 18,30 (2020).

[7] T.S. Orlova, A.M. Mavlyutov, M.Y. Gutkin. Mater. Sci. Eng. A 802, 140588 (2021).

[8] A.P. Zhilyaev, T.G. Langdon. Prog. Mater. Sci. 53, 893 (2008).

[9] G.K. Williamson, R.E. Smallman. Phil. Mag. 1, 34 (1956).

[10] Дж.Э. Хетч. Алюминий. Свойства и физическое металловедение. Металлургия, М. (1989). 422 с.

[11] T.S. Orlova, A.M. Mavlyutov, A.S. Bondarenko, I.A. Kasatkin, M.Y. Murashkin, R.Z. Valiev. Phil. Mag. 96, 23, 2429 (2016).

[12] R.Z. Valiev, M.Yu. Murashkin, I. Sabirov. Sci. Mater. 76, 13 (2014). 
[13] A.M. Mavlyutov, A.S. Bondarenko, M.Yu. Murashkin, E.V. Boltynjuk, R.Z. Valiev, T.S. Orlova. J. Alloys Compd. 698, 539 (2017).

[14] T.S. Orlova, T.A. Latynina, A.M. Mavlyutov, M.Y. Murashkin, R.Z. Valiev. J. Alloys Compd. 784, 41 (2019).

[15] T.A. Latynina, A.M. Mavlyutov, M.Yu. Murashkin, R.Z. Valiev, T.S. Orlova. Phil. Mag. 99, 19, 2424 (2019).

[16] А.М. Мавлютов, И.А. Касаткин, М.Ю. Мурашкин, Р.3. Валиев, Т.С. Орлова. ФТТ 10, 1998 (2015).

[17] A.M. Mavlyutov, T.S. Orlova, T.A. Latynina, I.A. Kasatkin, M.Y. Murashkin, R.Z. Vaiev. Rev. Adv. Mater. Sci. 52, 61 (2017).

[18] R.W Hertzberg. Deformation and fracture mechanics of engineering materials. Wiley, N.Y. (1989). 680 p.

[19] E.O. Hall. Proc. Phys. Soc. B 64, 747 (1951).

[20] N.J. Petch. Acta Crystallographica 6, 1, 96 (1953).

[21] N. Kamikawa, X. Huang, N. Tsuji, N. Hansen. Acta Mater. 57, 14, 4198 (2009).

[22] N. Hansen, X. Huang. Acta Mater. 46, 1827 (1998).

[23] F.R.N. Nabarro, Z.S. Basinski, D.B. Holt. Adv. Phys. 13, 193 (1964).

[24] И.И. Новиков. Металлургия, М. (1975). 208 с.

[25] O.R. Myhr, O. Grong, S.J. Andersen. Acta Mater. 49, 65 (2001).

[26] G.J. Mahon, G.J. Marshall. JOM 48, 6, 39 (1996).

[27] L.M. Brown, R.K. Ham. Strengthening methods in crystals. London: Appl. Sci. (1971).

[28] P.B. Hirsch, F.J. Humphreys. Physics of Strength and Plasticity. AS Argon (1969).

[29] H. Asgharzadeh, A. Simchi, H.S. Kim. Mater. Sci. Eng. A 528, 12, 3981 (2011).

[30] H. Asgharzadeh, A. Simchi, H.S. Kim. Metallurg. Mater. Transact. A 42, 3, 816 (2011).

[31] T.D. Topping, B. Ahn, Y. Li, S.R. Nutt, E.J. Lavernia. Metallurg. Mater. Transact. A 43, 2, 505 (2012).

[32] P.L. Rossiter. The Electrical Resistivity of Metals and Alloys. Cambridge University Press, Cambridge (2003). 452 p.

[33] Y. Miyajima, S.Y. Komatsu, M. Mitsuhara, S. Hata, H. Nakashima, N. Tsuji. Phil. Mag. 90, 4, 4475 (2010).

[34] J. Komatsu. Technical specification on hardenability of Boron Steel. N. KES (KNOW HOW). 7, 216, 2.

[35] A.S. Karolik, A.A. Luchvich. J. Phys.: Condens. Matter. 6, 873 (1994).

[36] Physical Metallurgy / Eds R.W. Cahn, P. Haasen. NorthHolland Publ. Co. Amsterdam (1983).

[37] M. Mito, H. Matsui, T. Yoshida, T. Anami, K. Tsuruta, H. Deguchi, T. Iwamoto, D. Terada, Y. Miyajima, N. Tsuji. Rev. Sci. Instrum. 87, 5, 053905 (2016).

[38] Y. Nasedkina, X. Sauvage, E.V. Bobruk, M.Y. Murashkin, R.Z. Valiev, N.A. Enikeev. J. Alloy Comp. 710, 736 (2017).

[39] G. Nurislamova, X. Sauvage, M. Murashkin, R. Islamgaliev, R.Z. Valiev. Phil. Mag. Lett. 88, 6, 459 (2008).

Редактор Д.В. Жуманов 